\title{
MIGRAÇÃO MÍNIMOS QUADRADOS (LSM) USANDO OPERADORES DE MIGRAÇÃO E MODELAGEM TIPO KIRCHHOFF
}

\author{
Peterson Nogueira Santos, INCT-GP/CNPq, Reynam C. Pestana, CPGG/UFBA
}

Copyright 2014, SBGf - Sociedade Brasileira de Geofísica.

Este texto foi preparado para a apresentação no VI Simpósio Brasileiro de Geofísica, Porto Alegre, 14 a 16 de outubro de 2014. Seu conteúdo foi revisado pelo Comitê Técnico do VI SimBGf, mas não necessariamente representa a opinião da SBGf ou de seus associados. É proibida a reprodução total ou parcial deste material para propósitos comerciais sem prévia autorização da SBGf.

\begin{abstract}
In this work we propose a method based on the theory of least square migration (LSM) for prestack datasets. Operators based on the Kirchhoff method were used for modeling and migration and during the demigration/modeling procedure the Born approximation was applied. The method implemented and tested is a modeling and migration Kirchhoff type (LSKM) combined with the LSM. The results presented allows one to show a better reconstitution of the reflectivity model and migrated image with higher resolution in comparison with the conventional Kirchhoff result.
\end{abstract}

\section{Introdução}

A migração Kirchhoff tem sido bastante empregada no processamento sísmico devido ao seu baixo custo computacional. Porém os algoritmos do tipo Kirchhoff são implementados usando aproximação de alta frequência e assim não incluem todos os efeitos modelados pela equação da onda que permitem a formação da imagem abaixo de fortes contrastes laterais de velocidade, com geometria irregular como domo de sal (Moreira Neto, 2004).

Os métodos de otimização da imagem migrada vêm ganhando espaço na indústria do petróleo com vista a aumentar a resolução da imagem resultante da migração. Neste trabalho, o método implementado foi o LSM, que consiste em buscar um modelo que seja o mais próximo possível do modelo de refletividade. Para cada modelo obtido, geralmente de forma iterativa, modelam-se dados sintéticos (calculados), que são comparados com os dados reais (observados). O modelo final será aquele onde o erro entre os dados observados e os dados calculados seja mínimo.

Segundo Oliveira (2014), um operador de migração pode ser considerado como o adjunto de um operador de modelagem sísmica direta, semelhante ao utilizado nas iterações da inversão completa da forma de onda. Embora o operador adjunto de migração convencional $\left(\mathbf{L}^{T}\right)$ seja uma razoável aproximação do operador inverso de modelagem direta $\left(\mathbf{L}^{-1}\right)$, ele não representa um operador inverso exato, mas pode ser 'adaptado' para uma aproximação do mesmo. De fato, na maioria dos casos, os dados sísmicos não podem ser otimizados de modo que o modelo da refletividade seja recuperado. Essa limitação deve-se ao fato de que as equações de modelagem não são suficientemente consistentes e os dados são registrados com um ângulo de iluminação limitado. Uma alternativa para se contornar o problema é aproximar o operador inverso exato por um operador inverso generalizado que pode ser formulado como um operador de migração por mínimos quadrados (LSM), (Nemeth, Wu e Schuster, 1999). A operação realizada por LSM é equivalente a uma inversão linearizada da função refletividade para uma determinada distribuição de velocidade.

A técnica LSM foi aplicada utilizando o método gradiente para otimizar a cada iteração a imagem migrada. Neste trabalho, o operador de modelagem foi construído a partir da solução da equação acústica da onda. O cálculo deste operador apresentado aqui é baseado na aproximação de Born dos campos de pressão associados ao modelo de velocidade e desse modo calcula-se o operador adjunto ao de modelagem, o operador de migração.

\section{Método LSKM pré-empilhamento no domínio do tiro}

Assumindo que o processo de modelagem seja linear, ou seja, (Nemeth, Wu e Schuster, 1999):

$$
\mathbf{d}=\mathbf{L m}
$$

onde $\mathbf{L}$ é o operador linear de modelagem, d é o dado modelado e m é modelo de pseudo-refletividade. Segundo Dai, Fowler e Schuster (2012) pode-se escrever o operador de modelagem Kirchhoff como:

$$
\mathbf{L}=\omega^{2} \mathbf{m}\left(\mathbf{r}^{\prime}\right) W(\omega) \mathbf{G}_{\mathbf{0}}\left(\mathbf{r}^{\prime}, \mathbf{r}_{\mathbf{s}}\right) \mathbf{G}_{\mathbf{0}}\left(\mathbf{r}, \mathbf{r}^{\prime}\right)
$$

onde $\mathbf{G}_{\mathbf{0}}\left(\mathbf{r}^{\prime}, \mathbf{r}_{\mathbf{s}}\right)$ e $\mathbf{G}_{\mathbf{0}}\left(\mathbf{r}, \mathbf{r}^{\prime}\right)$ são as funções de Green. O dado observado $\mathbf{d}_{o}$ é descrito por

$$
\mathbf{d}_{o}=\mathbf{L}_{o} \mathbf{m}_{o}
$$

e $\mathbf{m}_{o}$ é a refletividade verdadeira. Também assume-se que $\mathbf{L}=\mathbf{L}_{o}$. Na migração sísmica, usa-se a transposta do operador de modelagem Kirchhoff na equação (1) para obter:

$$
\mathbf{m}_{\text {mig }}=\mathbf{L}^{T} \mathbf{d}
$$

Segundo Dai, Fowler e Schuster (2012), $\mathbf{m}_{\text {mig }}$ é o resultado da migração Kirchhoff do dado modelado. Além disso, o operador de migração Kirchhoff $\left(\mathbf{L}^{T}\right)$ é descrito da seguinte maneira:

$$
\mathbf{L}^{T}=\sum_{r_{s}} \omega^{2} W^{*}(\omega) \mathbf{P}_{1} \mathbf{G}_{\mathbf{0}}^{*}\left(\mathbf{r}^{\prime}, \mathbf{r}_{\mathbf{s}}\right) \mathbf{G}_{\mathbf{0}}^{*}\left(\mathbf{r}, \mathbf{r}^{\prime}\right)
$$

em que $\mathbf{m}_{\text {mig }}$ é a seção migrada. Substituindo-se a equação (1) na equação (4) resulta em:

$$
\mathbf{m}_{m i g}=\mathbf{L}^{T} \mathbf{L m}
$$


A matriz $\mathbf{L}^{T} \mathbf{L}$ é a Hessiana e define $\mathbf{m}_{\text {mig }}$ como uma versão filtrada de $\mathbf{m}$ por $\mathbf{L}^{T} \mathbf{L}$. Assim, a migração sísmica irá reconstituir a refletividade $\mathbf{m}$, se $\mathbf{L}^{T} \mathbf{L}$ for uma matriz identidade. Em muitos casos $\mathbf{L}^{T} \mathbf{L}$ não é uma matriz identidade (Nemeth, Wu e Schuster,1999).

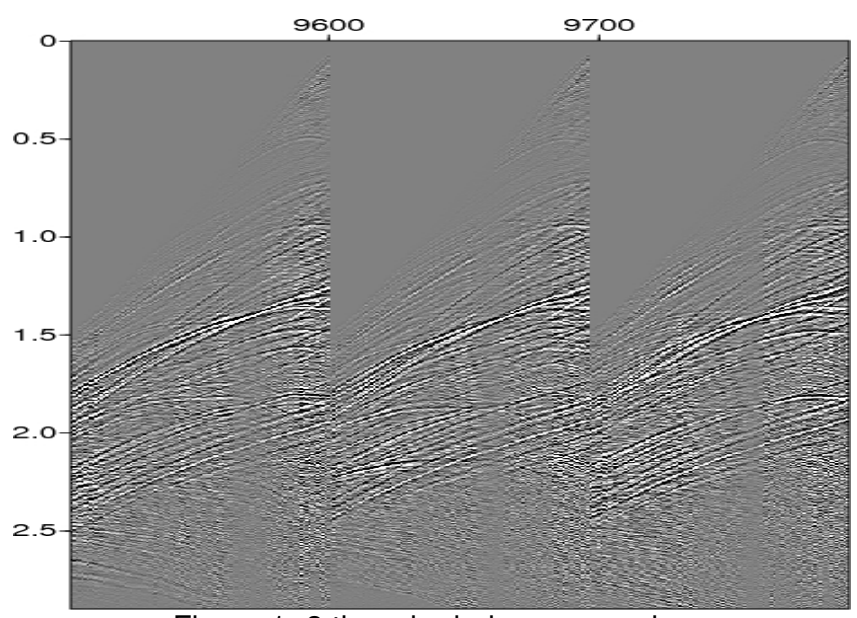

Figura 1: 3 tiros do dado marmousi

Portanto, segundo Oliveira (2014), o operador de migração sísmica pode ser considerado como o adjunto do operador de modelagem sísmica, como usado nos processos de inversão. Entretanto, o operador adjunto é uma boa aproximação para a inversa do operador de modelagem, mas ele não é a sua inversa exata. Porém, a migração sísmica pode ser implementada para tentar aproximar o operador inverso exato. Para isso, podemos nos apropriar do método dos mínimos quadrados e resolver esse problema através de um método iterativo. Neste trabalho, foi usado o método gradiente (steepest descent). Pode-se obter a solução, minimizando a função objetivo $O(\mathbf{m})$, que é definida como os mínimos quadrados da diferença entre o dado modelado e o dado observado, ambos no domínio do tempo, que é dada por:

$$
O(\mathbf{m})=\left\|\mathbf{L m}-\mathbf{d}_{o}\right\|^{2}
$$

O esquema numérico abaixo, mostra o cálculo da solução mínimos quadrados:

$$
\begin{gathered}
\mathbf{G}_{k}=\mathbf{L}^{T}\left[\mathbf{L} \mathbf{m}-\mathbf{d}_{o}\right] \\
\alpha_{k}=\frac{\mathbf{G}_{k}^{T} \mathbf{G}_{k}}{\mathbf{E}_{k}^{T} \mathbf{E}_{k}} \\
\mathbf{m}_{k+1}=\mathbf{m}_{k}-\alpha_{k} \mathbf{G}_{k}
\end{gathered}
$$

A equação (8) representa a migração do erro entre o dado calculado e o dado observado, associado ao modelo $\mathbf{m}$. Esse vetor de resíduos migrado está na mesma direção do vetor gradiente definido em $\mathbf{m}$, porém em sentido oposto (Porsani, 2008). O vetor gradiente é representado por $\mathbf{G}_{k}$, e matematicamente, esse vetor aponta na direção de máxima inclinação. O parâmetro $\alpha_{k}$ representa o tamanho do passo a cada iteração feita na otimização e $k$ é o número da iteração. A equação (10) é o resultado da migração da imagem otimizada, na qual é atualizada para cada iteração.

\section{Resultados}

Nesta seção apresenta-se os resultados do método LSKM para o modelo Marmousi. Dentro do processo de otimização da imagem foi preciso fazer alguns testes para se obter uma imagem com uma melhor resolução, no qual o principal teste foi obter o parâmetro $\left(\alpha_{k}\right)$ pelo método gradiente adaptado, que corresponde ao tamanho do passo a ser executado pelo vetor gradiente $\left(\mathbf{G}_{k}\right)$ para atualizar o modelo corrente. Entretanto, neste método gradiente foi economizado no processo de otimização uma demigração, pois no cálculo do parâmetro $\left(\alpha_{k}\right)$ foi usado a matriz dos resíduos em vez do método gradiente convencional que usa a demigração do gradiente. Esta adaptação é conveniente, pois a matriz dos resíduos é o dado sísmico de entrada na migração para obter o gradiente. Sendo assim, demigrar o gradiente é equivalente a obter a matriz dos resíduos novamente.

Como neste trabalho foi utilizada a técnica de traçamento de raios, o campo de velocidade foi suavizado de maneira a se obter uma maior densidade de raios na região do corpo de sal e sua vizinhança.

A partir dos resultados, verificou-se que o método proposto aqui conseguiu imagear melhor os refletores em todo o modelo, delineando melhor as estruturas, na qual a migração Kirchhoff convencional não conseguiu iluminar.

Desse modo, obteve-se imagens otimizadas até 50 iterações. A seguir são mostrados o campo de velocidade original (Figura 2), o suavizado (Figura 3) e algumas seções otimizadas (Figura 5 até Figura 11).

$\mathrm{Na}$ Figura 4, é o resultado da migração Kirchhoff convencional que será usado para iniciar o processo de inversão.

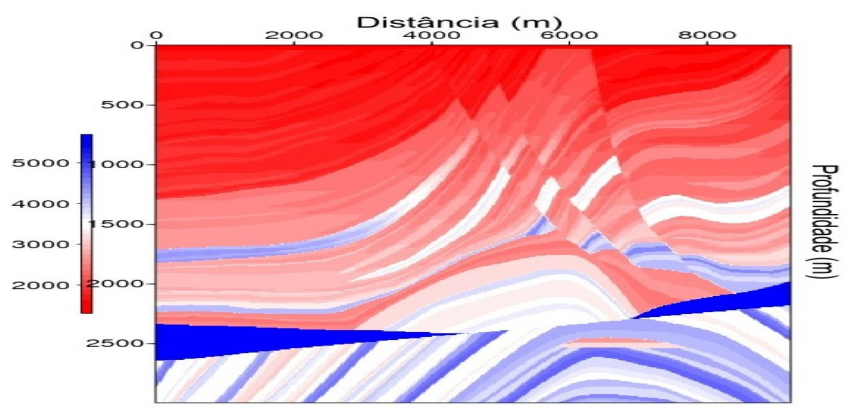

Figura 2: Campo de velocidade do modelo Marmousi

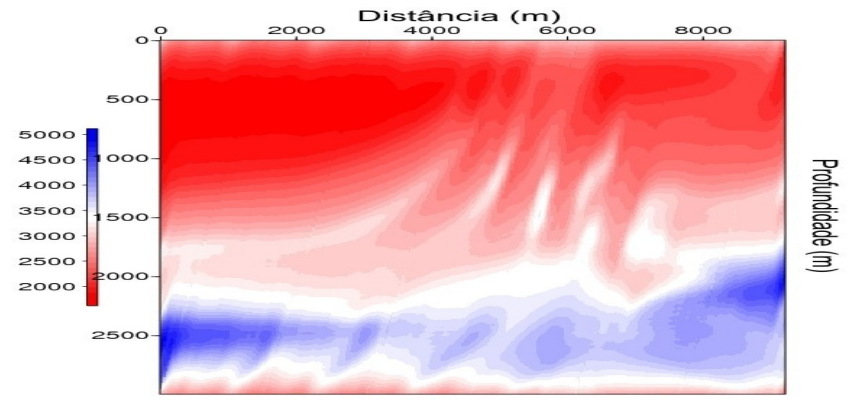

Figura 3: Campo de velocidade suavizado do modelo Marmousi 


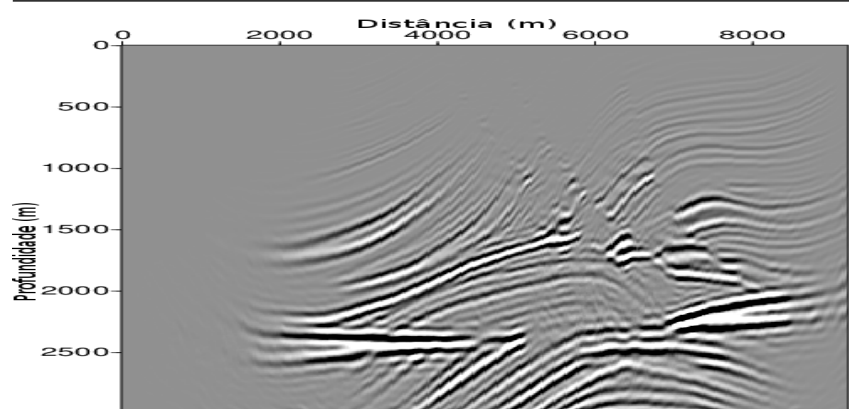

Figura 4: Migração Kirchhoff convencional do modelo Marmousi $\left(\mathbf{m}_{\mathbf{0}}\right)$

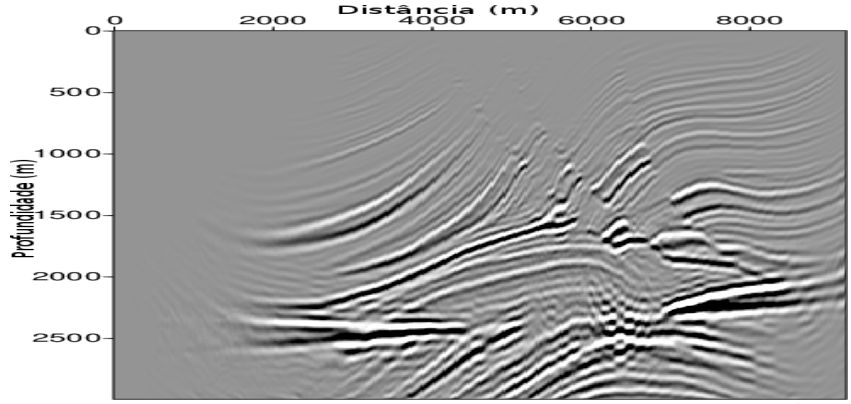

Figura 5: Seção LSKM do modelo Marmousi préempilhamento no domínio do tiro com atualização da imagem para 5 iterações

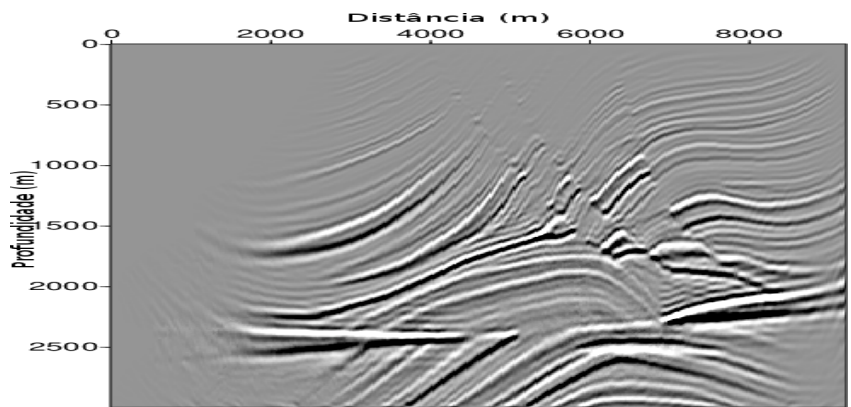

Figura 6: Seção LSKM do modelo Marmousi préempilhamento no domínio do tiro com atualização da imagem para 10 iterações

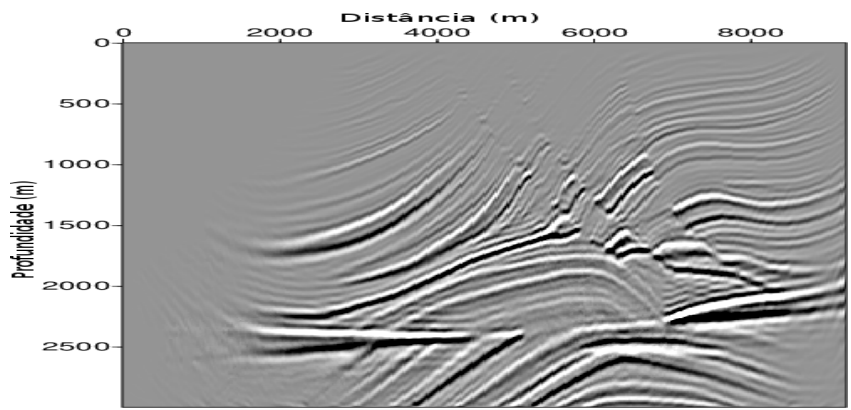

Figura 7: Seção LSKM do modelo Marmousi préempilhamento no domínio do tiro com atualização da imagem para 15 iterações

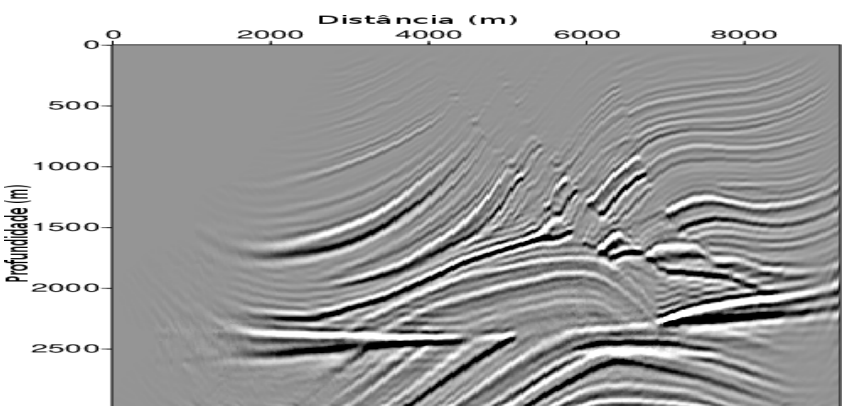

Figura 8: Seção LSKM do modelo Marmousi préempilhamento no domínio do tiro com atualização da imagem para 20 iterações

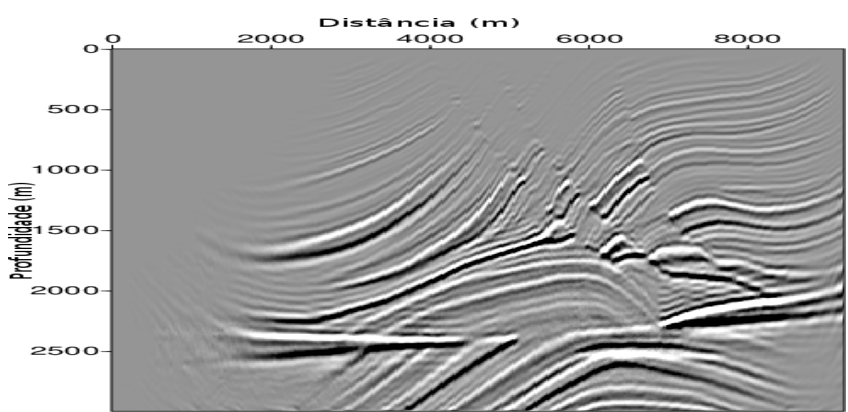

Figura 9: Seção LSKM do modelo Marmousi préempilhamento no domínio do tiro com atualização da imagem para 30 iterações

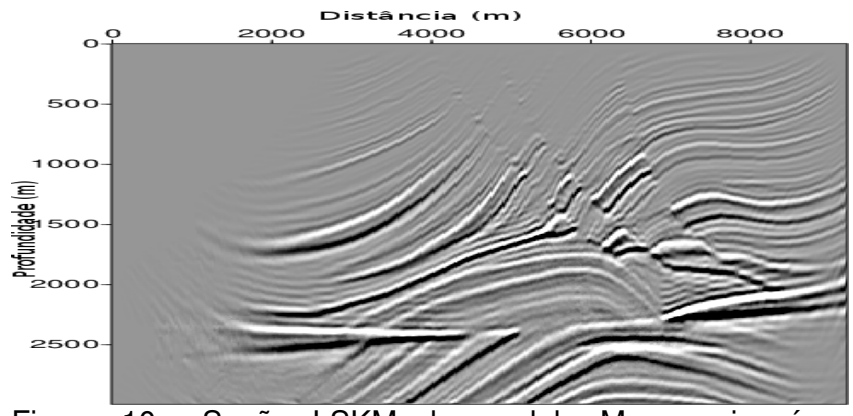

Figura 10: Seção LSKM do modelo Marmousi préempilhamento no domínio do tiro com atualização da imagem para 40 iterações

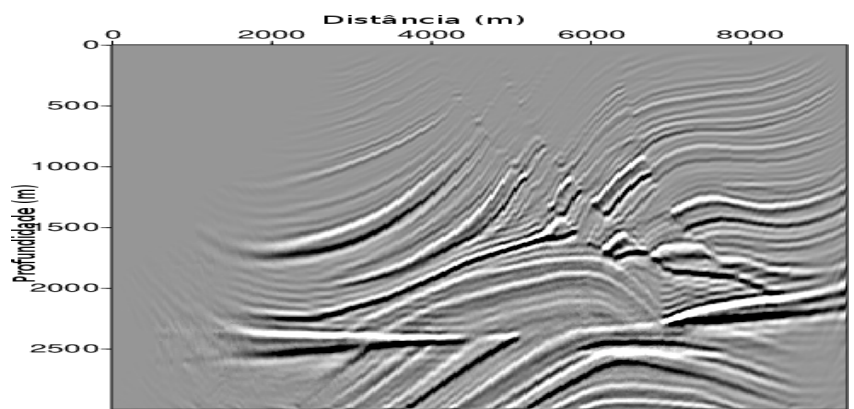

Figura 11: Seção LSKM do modelo Marmousi préempilhamento no domínio do tiro com atualização da imagem para 50 iterações 


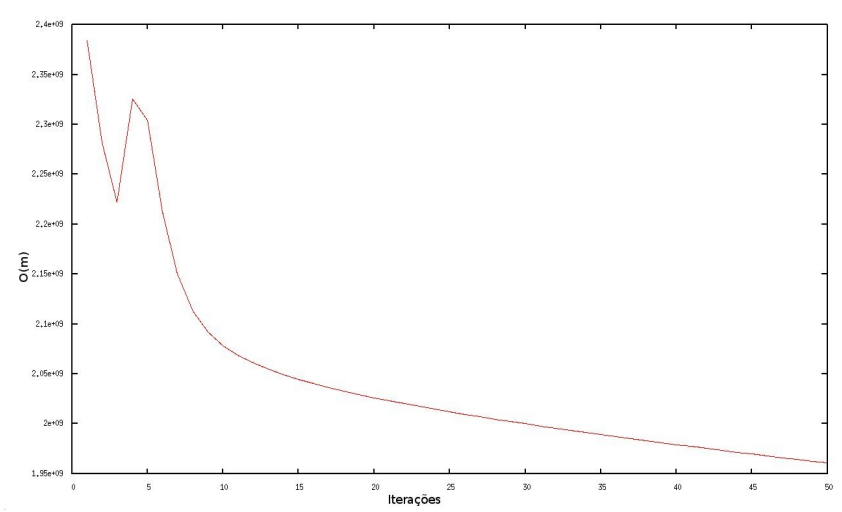

Figura 12: Gráfico que mostra o comportamento de $O(m)$ em função das iterções correntes do modelo marmousi

\section{Conclusões}

Como foi verificado na seção anterior, os resultados apresentados pelo método LSKM pré-empilhamento em profundidade, baseados na solução mínimos quadrados, produziram seções migradas de boa qualidade atestando a capacidade desse método em obter resultados de melhor resolução.

Os resultados obtidos com essa metodologia de otimização, conseguiram reconstituir a seção refletividade, mostrando imagens migradas de melhor qualidade do que a migração Kirchhoff convencional. Verificouse também como o método LSKM desenvolvido se comporta na presença de falhas, dobras e armadilhas de hidrocarbonetos. $E$ foi possível constatar que a técnica de migração usando operadores Kirchhoff imageou com sucesso as estruturas geológicas, reconstituindo a seção refletividade.

No que diz respeito a função objetivo, observa-se que houve uma diminuição da função $(O(m))$ em relação ao número de iterações. Ou seja, a função objetivo convergiu para o valor mínimo, comprovando a eficiência do método.

O método LSM mostra-se bastante promissor como uma ferramenta para o imageamento sísmico. Ele tem despertado um crescente interesse da comunidade geofísica, o que justifica o grande número de publicações com diferentes implementações nos últimos anos (Oliveira, 2014). Portanto, fica evidente que o método de otimização baseado na solução dos mínimos quadrados é uma importante ferramenta que pode auxiliar no melhoramento da imagem diminuindo as dúvidas geradas pelos métodos convencionais de imageamento. No caso do LSKM, pode ser aplicado nas bacias brasileiras terrestres, na qual não possui tectônica salífera, uma vez que o método não é indicado para regiões com fortes contrastes de velocidades.

\section{Agradecimentos}

Os autores agradecem ao CPGG/UFBA/INCT-GP, pelo apoio no desenvolvimento deste trabalho. Peterson Nogueira Santos agradece ao CNPq/INCT-GP pelo suporte através da bolsa de pesquisa.

\section{Referências}

Costa, M. (1997) Migração de dados sísmicos 2-d ordenados em afastamento comum, Migração de dados sísmicos 2-D ordenados em afastamento comum.

Dai, W.; Fowler, P. e Schuster, G. T. (2012) Multisource least-squares reverse time migration, Geophysical Prospecting, 60(4):681-695.

Moreira Neto, C. (2004) Migração pré-empilhamento em profundidade utilizando ondas planas, Migração préempilhamento em profundidade utilizando ondas planas.

Nemeth, T.; Wu, C. e Schuster, G. T. (1999) Least-squares migration of incomplete reflection data, Geophysics, 64(1):208-221.

Nogueira Santos, P.; Pestana, R.; Santos, A. W. G. e Aldunate, G. (2013) Migração kirchhoff 2d pósempilhamento otimizada através do método dos mínimos quadrados, sociedade Brasileira de Geofísica.

Oliveira, A. (2014) Migração reversa no tempo (rtm) com compensação de iluminação no domínio de ondas planas e por mínimos quadrados (Ism) no domínio do tiro, Migração Reversa no Tempo (RTM) com Compensação de lluminação no Domínio de Ondas Planas e por Mínimos Quadrados (LSM) no Domínio do Tiro.

Porsani, M. (2008) O método dos mínimos quadrados, O método dos mínimos quadrados.

Romanelli, A. (2010) Análise do sinal sísmico, Sociedade Brasileira de Geofísica (SBGf). Rio de Janeiro.

Schuster, G. T. et al. (1993) Least-squares cross-well migration, In: 1993 SEG Annual Meeting, Society of Exploration Geophysicists.

Yilmaz, O. (2001) Seismic data analysis, vol. 1, Society of Exploration Geophysicists Tulsa. 